\title{
Desdobramentos do Pensamento Computacional no Brasil *
}

\author{
Adriana Bordini ${ }^{1}$, Christiano Avila ${ }^{1}$, Mônica Cunha ${ }^{1}$, \\ Simone Cavalheiro ${ }^{1}$, Luciana Foss ${ }^{1}$ \\ ${ }^{1}$ Centro de Desenvolvimento Tecnológico - Universidade Federal de Pelotas (UFPel) \\ Rua Gomes Carneiro, 1 - 96.010-610 - Pelotas - RS - Brasil \\ \{adriana.bordini, cm.avila,mmd.cunha, simone.costa\}@inf.ufpel.edu.br
}

\begin{abstract}
This article presents a survey of projects in the field of Computational Thinking (CT), which had published results in the main vehicles about Education and Computation in Brazil. This study identified seven approaches that have been adopted for the introduction of the CT in Brazil, of which the most frequent are the introduction of CT with different target audiences and proposals or assessments of methodologies and practices to develop CT. The research was done by addressing the questions: What has been the main goals of the projects in the area? What has been the target audience and the number of research participants? What are the results reported? What tools have been adopted? The goal of this survey is to describe the developments in Brazil in the CT subject.
\end{abstract}

Resumo. Este artigo apresenta um levantamento de projetos na área do Pensamento Computacional (PC) que foram publicados nos principais veículos de Informática na Educação no Brasil. Neste estudo foram identificadas 7 abordagens que têm sido adotadas para a introdução do PC no Brasil, das quais as de maior ocorrência são a introdução de conceitos do PC com diferentes públicoalvo e propostas ou avaliações de metodologias e práticas para desenvolver $o$ PC. A pesquisa foi realizada, procurando responder as seguintes questões: Qual tem sido o objetivo de estudo dos trabalhos na área? Qual o público alvo e o número de participantes da pesquisa? Quais os resultados reportados? Quais ambientes ou ferramentas têm sido adotados? O objetivo deste levantamento é descrever os desdobramentos no Brasil sobre o tema PC.

\section{Introdução}

Pensamento Computacional (PC), segundo [Wing 2006] envolve a resolução de problemas, projetos de sistemas e a compreensão do comportamento humano, recorrendo aos conceitos fundamentais da ciência da computação. Argumenta-se também que o Pensamento Computacional é uma habilidade básica a ser desenvolvida em todas as crianças em idade escolar, assim como ler, escrever e fazer as operações aritméticas básicas. Neste sentido, o estudo do PC é de grande importância para toda a sociedade, pois são conhecimentos adquiridos para a vida toda, tanto pessoal quanto profissional, que auxiliam na busca de soluções de problemas, em qualquer área [Wing 2006].

Nesta pesquisa foram identificados alguns trabalhos que fazem análises sobre o PC ou discutem alguns desafios para a sua implantação [Raabe et al. 2015,

${ }^{*}$ Projeto realizado com o apoio do PROEXT - MEC/SESu 
V Congresso Brasileiro de Informática na Educação (CBIE 2016)

Anais do XXVII Simpósio Brasileiro de Informática na Educação (SBIE 2016)

Carvalho et al. 2013]. Dos levantamentos já realizados, encontra-se um estudo sobre as principais ferramentas que têm sido utilizadas no ensino-aprendizagem do PC [Bombasar et al. 2015], um trabalho sobre as habilidades envolvidas [Farias et al. 2015] e uma pesquisa sobre o conhecimento do termo PC [Araújo et al. 2015]. Também encontram-se trabalhos que investigam as relações da Matemática com o PC [Barcelos and Silveira 2012, Barcelos et al. 2015, Mestre et al. 2015]. Alguns projetos sobre PC que focam na Educação Básica são descritos em [Weisshahn et al. 2015].

Além disso, esforços têm sido feitos no sentido de disseminar e introduzir o PC no Brasil [Rodriguez et al. 2015, França and Tedesco 2014]. Diferente dos levantamentos já realizados, este trabalho objetiva compilar estes esforços e obter um panorama geral dos desdobramentos da área neste tema. Para isso, fez-se um levantamento de artigos publicados na área do PC no Brasil, objetivando sintetizar as seguintes questões: Qual tem sido o objetivo de estudo dos trabalhos na área? Qual o público alvo e o número de participantes da pesquisa? Quais ambientes ou ferramentas têm sido adotados? Quais os resultados reportados?

Este artigo traz um levantamento de artigos dos últimos 6 anos que objetivam disseminar o PC no Brasil. O trabalho está organizado como segue. A seção 2 detalha a metodologia utilizada no mapeamento realizado. A seção 3 descreve o panorama geral dos trabalhos na área do PC no Brasil e a seção 4 contém as considerações finais.

\section{Metodologia de Busca}

O levantamento de projetos na área do PC no Brasil considerou os artigos publicados nos últimos 6 anos em periódicos e anais de conferências brasileiros, abrangendo o período de 2010 a 2015. Considerou-se para esta pesquisa os principais veículos da área de Informática na Educação no Brasil: SBIE (Simpósio Brasileiro de Informática na Educação), WIE (Workshop de Informática na Escola), WAlgProg (Workshop de Ensino em Pensamento Computacional, Algoritmos e Programação), RBIE (Revista Brasileira de Informática na Educação), WEI (Workshop sobre Educação em Computação), WEIT (Workshop-Escola de Informática Teórica), RENOTE (Revista Novas Tecnologias na Educação) e DesafIE (Workshop de Desafios da Computação Aplicada à Educação).

A pesquisa foi realizada nos sites de busca dos veículos, onde se considerou apenas os trabalhos que focam na área do PC. A busca foi feita pelos termos "Pensamento Computacional", "Raciocínio Computacional" ou "Computational Thinking", trazendo um total de 45 publicações. A Tabela 1 sintetiza os trabalhos considerados nesta análise. Dentre os trabalhos analisados, identificou-se que sete diferentes objetivos têm sido buscados ${ }^{1}$ : introdução de conceitos do PC (denotado por INT na Tabela); proposta ou avaliação de metodologias (MET); investigação, apresentação ou avaliação de ferramentas para o ensino de computação no ensino básico (FER); atração de jovens para a área de computação (ATR); desafios de implantação e levantamentos sobre PC (LEV); formação de professores (FOR); e correlação entre as competências de diretrizes nacionais e internacionais com o PC (COR). Foram identificados, em relação ao público-alvo, diferentes focos de atuação: Ensino Fundamental (EF), Ensino Médio (EM), Educação Básica (EB)

\footnotetext{
${ }^{1}$ A categorização foi realizada de acordo com o foco do trabalho. Por exemplo, considerando o Scratch, trabalhos que o adotam para o ensino de programação estão em INT, os que o propõe como uma metodologia de ensino, estão em MET e trabalhos que analisam seus recursos e funcionalidades, encontram-se em FER.
} 
V Congresso Brasileiro de Informática na Educação (CBIE 2016)

Anais do XXVII Simpósio Brasileiro de Informática na Educação (SBIE 2016)

que envolve alunos do EF e EM, Ensino Técnico (ET), Ensino Superior (ES), Professores (PROFE) e Profissionais (PROFI). Alguns trabalhos não envolvem público alvo e são denotados por NA na Tabela (indicando que o item não se aplica).

O mapeamento do estudo realizado foi organizado procurando responder as seguintes questões de pesquisa: QP1 - Qual tem sido o objetivo de estudo dos trabalhos na área? QP2 - Qual o público alvo e o número de participantes da pesquisa? QP3 - Quais ambientes ou ferramentas têm sido adotados? QP4 - Quais os resultados reportados?

\section{Pensamento Computacional no Brasil}

Nesta seção apresenta-se um resumo geral das respostas obtidas para as questões de pesquisa consideradas. Para isso, foi feito uma categorização e agrupamento prévio dos 45 artigos incluídos neste estudo, para posterior análise detalhada dos itens considerados. Esta categorização considerou os objetivos principais dos trabalhos. Vale lembrar que alguns artigos estão em mais de uma categoria.

\subsection{Qual tem sido o objetivo de estudo dos trabalhos na área do PC no Brasil?}

Introdução de Conceitos Fundamentais do PC: dos 45 artigos, 20 (identificados com ID do 1 ao 20 da Tabela 1), relatam experiências de projetos que visam introduzir conceitos fundamentais do PC [CSTA 2011] e que são efetivados, em geral, por meio de cursos, minicursos ou oficinas de linguagens ou ambientes de programação e/ou robótica.

O objetivo destes cursos é proporcionar o aprendizado dos conceitos relacionados à lógica de programação por meio de algoritmos, a introdução de conceitos de computação através de atividades desplugadas [Bell et al. 1998], a utilização de linguagens/ambientes de programação, a implementação de atividades relacionadas à robótica educacional ou, ainda, a combinação de duas ou mais atividades. Os trabalhos classificados nesta categoria buscam desenvolver nos aprendizes as habilidades do pensamento computacional como, por exemplo, desenvolvimento do raciocínio lógico, capacidade de formulação e resolução de problemas, promoção da autonomia e da criatividade, dentre outras.

Proposta ou avaliação de metodologias e práticas para desenvolver o $\mathbf{P C}^{2}$ : um outro grupo de artigos (com ID do 19 ao 28 da Tabela 1) possui como objetivo desenvolver ou avaliar propostas de metodologias ou práticas para trabalhar com o pensamento computacional. Alguns trabalhos se baseiam na premissa da aprendizagem significativa, delineando uma estratégia de ensino de modo contextualizado. A teoria da aprendizagem significativa é representada pela interação cognitiva existente entre um conhecimento prévio e um novo conhecimento. Dessa forma, ela ocorre em "qualquer atividade na qual a representação cognitiva de experiência prévia e os componentes de uma situação problemática apresentada são reorganizados a fim de atingir um determinado objetivo" (Ausubel, 1968 apud [Ramos and Teixeira 2015]).

Em outro trabalho, propõe-se a adoção do Scratch para a exposição de conceitos, práticas computacionais e a avaliação da aprendizagem. Outra metodologia sugerida se baseia na autorregulação, onde os alunos definem seus próprios objetivos, monitoram e

\footnotetext{
${ }^{2}$ Nesta categoria, metodologias são consideradas (combinação de) trajetórias que direcionam o processo de ensino-aprendizagem do PC. Práticas são as ações adotadas nestas trajetórias que propiciam o aluno ser um sujeito ativo na construção do conhecimento.
} 
V Congresso Brasileiro de Informática na Educação (CBIE 2016)

Anais do XXVII Simpósio Brasileiro de Informática na Educação (SBIE 2016)

avaliam a própria aprendizagem. O modelo denominado PenC, baseia-se nesta proposta e possui 4 fases: pré-reflexão, resolução, avaliação por pares e pós-reflexão.

Outros trabalhos abordam métodos de desenvolvimento do PC, considerando o escopo dos cursos de Licenciatura em Computação. O método ABILSEN (Abordagem de Inclusão do Licenciado em Computação no Ensino Básico), possui como objetivo possibilitar que o Licenciado em Computação possa atuar, de forma transversal, em conjunto com os professores das demais disciplinas, tais como, Matemática, Geografia e Física. O método teve como base para sua criação o ciclo PDCA (plan, do, check e action), e possui as seguintes etapas: P - definição do ambiente, planejamento da disciplina e seleção dos objetos de aprendizagem; D - aplicação com mediação e desenvolvimento dos objetos de aprendizagem; C - avaliação da aprendizagem e A - refinamento de práticas.

O método de tutoria, envolvendo computação desplugada e robótica, é também proposto para ser adotado com alunos que estão sendo introduzidos à programação de computadores. Particularmente, a abordagem foi motivada pelo alto índice de evasão nas disciplinas de programação de cursos de Licenciatura em Computação.

A robótica educacional livre (com o uso de placas Arduino e do software Scratch) também é uma estratégia proposta para amenizar as dificuldades de iniciantes em programação. Robótica Educacional ou Pedagógica Livre [Cesar 2013] é um conjunto de processos e procedimentos envolvidos em propostas de ensino e de aprendizagem que utilizam kits pedagógicos, baseados em soluções livres e em sucatas como tecnologia de mediação para a construção do conhecimento, tudo isso focado no baixo custo.

Também já existe a proposta de um plano de ação para a promoção do PC nas escolas, o qual envolve as seguintes etapas: criação de uma comunidade de discussão; definição de estratégias para impactar as escolas; definição de uma agenda de pesquisa e produção e disseminação de material de orientação.

Papert e o construcionismo [Papert 1980] também são referências fundamentais destacadas na proposta de plano de ação do projeto DEMULTS (Desenvolvimento Educacional de Multimídias Sustentáveis) que introduz metodologias participativas no desenvolvimento de jogos, onde o usuário final não é apenas um jogador mas participa do processo de desenvolvimento (promovendo sua autonomia). Particularmente, o construcionismo defende que o computador na educação deve ser uma ferramenta para promover o pensamento crítico na solução de problemas.

Investigação, apresentação ou avaliação de ferramentas para o ensino de computação no ensino básico: os artigos com ID 29 ao 34 da Tabela 1 tiveram como objetivo estudar a efetividade de ferramentas para implantação de projetos na área do PC. As ferramentas RoboMind, Scratch, Linguagem Logo, Kodu Game Labs e Alice foram analisadas quanto a interface, usabilidade e fidelidade com conceitos da Computação.

Os dois métodos de avaliação, formativa e objetiva, foram utilizados na análise dos jogos Lightbot e The Foss. Na abordagem objetiva, um especialista avalia o software verificando um conjunto de critérios pré-definidos, enquanto a abordagem formativa investiga a interação entre alunos, professores e ferramenta através de observação de atividades e/ou entrevistas com os mesmos [Falcão and Barbosa 2015].

Já o jogo Computino foi criado para trabalhar conceitos de números binários com 
V Congresso Brasileiro de Informática na Educação (CBIE 2016)

Anais do XXVII Simpósio Brasileiro de Informática na Educação (SBIE 2016)

estudantes da educação básica. Também a tecnologia Tinta Digital foi utilizada para capturar o caminho percorrido por alunos na realização de uma tarefa, permitindo sua análise por um profissional ou de maneira automática, aplicando técnicas da inteligência artificial.

Atração de jovens para a área de computação: foram encontrados 7 trabalhos (ID 30 ao 36 da Tabela 1), que procuram atrair jovens em geral, ou especificamente meninas, para a área da computação. As estratégias utilizadas para isto são cursos de programação, desenvolvimentos de jogos e/ou aplicativos e participação em olimpíadas de programação.

Desafios de implantação e levantamentos sobre PC: objetivando realizar análises do PC, foram localizados 9 trabalhos (artigos com ID 28, 34, 37 ao 43 da Tabela 1). Um dos trabalhos investiga experiências didáticas que integram a Matemática e o PC, descrevendo particularmente, quais os conteúdos trabalhados, as ferramentas utilizadas e o público alvo. Uma outra pesquisa investiga se a área é conhecida e compreendida por profissionais, tanto da indústria quanto da academia.

Já uma revisão sistemática de literatura buscou identificar, no período de 2006 até o início de 2015, quais as linguagens de programação visuais são mais utilizadas e investigar o interesse da comunidade científica pela introdução do PC na educação básica. Além disso, um levantamento de oito projetos que aplicam PC na Educação Básica foi previamente realizado, com relação aos objetivos propostos, atividades realizadas e resultados alcançados. Por fim, alguns trabalhos discutem desafios a serem enfrentados para a implantação do PC na educação básica no Brasil.

Formação de professores ou multiplicadores do PC: os artigos com ID 41 e 44 da Tabela 1 se incluem nesta categoria. Os trabalhos identificam a necessidade de adequação da formação de multiplicadores em relação aos desafios do PC. Eles sugerem, além da realização de cursos e oficinas, que haja formação continuada para a prática docente, pois apenas $13,33 \%$ dos alunos concluintes de um curso de Lic. em computação relataram ter trabalhado no tema durante o curso.

Correlação entre as competências de diretrizes nacionais e internacionais com o PC: os artigos com ID 42, 43 e 45 da Tabela 1 têm por objetivo correlacionar as competências que podem ser desenvolvidas no PC com diretrizes nacionais ou internacionais. Uma correlação entre os parâmetros curriculares nacionais (PCN), especialmente relacionados com a matemática, com as competências a serem desenvolvidas com o pensamento computacional já foi previamente descrita. Também já foi analisada uma possível correlação entre o desempenho de alunos no ENEM e o conhecimento de programação de computadores. Além disso, foi identificado no teste de PISA (Programa Internacional de Avaliação de Estudantes) a existência de conceitos do PC nas questões de matemática.

\subsection{Qual o público alvo e a escala de aplicação das pesquisas sobre PC?}

Dentre os 45 trabalhos, 23 se destacam na abordagem experiências de ensino. Projetos nesta abordagem foram aplicados a diferentes públicos. Nesta questão, buscou-se descrever além do público alvo, a quantidade de alunos que participaram da pesquisa. Dentre estes 23 artigos, 18 trazem o público alvo e a escala de aplicação. Sendo 7 no EF, 7 no EM e 1 em cada uma das demais categorias (EB, ET, ES e PROFE).

Das experiências de ensino realizadas, a média de participação foi de 30 alunos no EF, 29 alunos no EM (com desvio padrão de 13.7 e 25.5 respectivamente). Nas de- 
V Congresso Brasileiro de Informática na Educação (CBIE 2016)

Anais do XXVII Simpósio Brasileiro de Informática na Educação (SBIE 2016)

mais categorias, houve apenas um relato de experiência, incluindo, 24 alunos na EB, 6 alunos no ET, 10 alunos no ES e 103 professores. Dentre estes trabalhos, experiências já foram realizadas com alunos do $1^{\circ}, 4^{\circ}, 5^{\circ}, 7^{\circ}, 8^{\circ}$ e $9^{\circ}$ anos do EF, com todos os anos do EM, com alunos do curso Técnico em Informática e com alunos do curso de Ciência da Computação. Apenas 5 trabalhos, que abordam experiência de ensino, não relatam a sua escala de aplicação, identificando apenas o público alvo (2 no EF, 1 no EM e 2 na EB).

\subsection{Quais ferramentas ou ambientes têm sido adotados em abordagens do PC?}

Dentre os 45 trabalhos selecionados, 37 adotam ferramentas ou ambientes na abordagem proposta. A Tabela 1 detalha um quadro resumo das ferramentas utilizadas, destacando para qual objetivo e qual público alvo elas têm sido adotadas.

O ambiente Scratch é a ferramenta mais utilizada em projetos de PC, aparecendo em 12 trabalhos, seguido da "Computação Desplugada", com 11 ocorrências, que aqui está sendo considerada como uma ferramenta para desenvolvimento do PC. O uso do Scratch, na sua maioria, são em experiências de ensino que trabalham conceitos fundamentais da computação para desenvolver o PC, em geral, são efetivados por meio de cursos e/ou oficinas que envolvem programação de computadores (algoritmos), animações e jogos. Já a "Computação Desplugada" é utilizada para trabalhar conceitos básicos da computação, como números binários, representação de imagens, conceitos de abstração, manipulação de dados, dentre outros propostos no livro [Bell et al. 1998].

E geral, os trabalhos que adotam ferramentas buscam além de apresentar os conceitos da computação, o desenvolvimento do raciocínio lógico, da capacidade de formulação e resolução de problemas e a promoção da autonomia e da criatividade.

\subsection{Quais os resultados reportados na área do $\mathrm{PC}$ no Brasil?}

Dentre os relatos de experiências de ensino, em sua grande maioria, os trabalhos reportam resultados positivos em relação a efetividade das ações na área do PC, indicando que os alunos tiveram desempenho satisfatório nas atividades e avaliações realizadas, que os jogos utilizados e/ou desenvolvidos facilitaram a compreensão dos conceitos da computação e que as atividades trabalhadas aumentaram o interesse pela área, entre outros.

Em apenas dois relatos foram apresentados resultados que não demonstraram melhoria no desempenho dos aprendizes nos testes realizados posteriormente à execução das atividades. Um trabalho que visava desenvolver habilidades necessárias para prática do PC em crianças do quarto ano do ensino fundamental, onde segundo os autores, este fato pode ter ocorrido por uma deficiência do processo de avaliação. Outro trabalho que também relata que não houve melhoria em relação as habilidades do PC, foca na aproximação de 22 meninas para a área de Computação. Os resultados mostraram que mais de $60 \%$ das alunas acertaram menos de $50 \%$ das questões da avaliação, considerado pela equipe como um desempenho ruim, já que os problemas eram considerados fáceis. Além disso, observou-se um alto índice (60\%) de desistência antes da conclusão do curso.

Os trabalhos que possuem como objetivo propor ou avaliar metodologias também reportam, em geral, que os alunos conseguiram acompanhar as atividades realizadas. Dos trabalhos que utilizaram o Scratch, de forma geral os autores concluem que o ambiente pode ser utilizado satisfatoriamente por estudantes da Educação Básica no desenvolvimento de habilidades e conceitos do PC, que o mesmo viabiliza a aprendizagem signifi- 
V Congresso Brasileiro de Informática na Educação (CBIE 2016)

Anais do XXVII Simpósio Brasileiro de Informática na Educação (SBIE 2016)

cativa e que os discentes gostam e avaliam positivamente as atividades nele realizadas. A partir da realização de oficinas Scratch com profissionais de escolas, relatou-se que $60 \%$ deles ficaram satisfeitos e os professores de física e matemática mostraram mais interesse.

Da abordagem que utiliza práticas com a robótica pedagógica relata resultados quantitativos satisfatórios, onde cerca de $75 \%$ pensam em trabalhar na área, e qualitativos onde os alunos acharam as aulas fáceis e divertidas, e a maioria deseja obter mais conhecimento sobre computação. A metodologia ABILSEN promoveu o interesse dos professores, os quais mostraram-se motivados a aplicar o método em suas disciplinas. Dos demais trabalhos, destaca-se a proposta de metodologias (PDCA, PenC, construcionismo, metodologia participativa e plano de ação) para a implantação do PC.

Dos artigos que tiveram por objetivo investigar, apresentar ou avaliar ferramentas para o ensino de computação no ensino básico, relata-se: RoboMind se mostra adequada para o ensino de conceitos de lógica de programação, mas se limita a estruturas de seleção e de repetição; Scratch é bastante interativa, mas falta um feedback passo a passo sobre o que o aluno produz na ferramenta; SuperLogo propicia a introdução da maioria dos conceitos de programação, mas a interação e o ambiente deixa a desejar no aspecto lúdico, uma vez que a interface é pouco atraente e interativa; Kodu Labs apresenta os conceitos de lógica de maneira lúdica e interativa, mas a limitação é a quantidade de personagens; Alice, ajuda na materialização de conceitos abstratos e introduz conceitos de orientação a objetos, mas possui diversos bugs e não explora o aspecto lúdico.

O jogo Computino despertou o interesse dos alunos e permitiu o desenvolvimento do conceito de números binários. O jogo The Foss foi avaliado como excelente em relação a afetividade, estruturação dos níveis do jogo e controle dos alunos; como bom quanto a facilidade de uso, design de tela, objetividade, papel do instrutor e motivação; e como regular quanto a forma que apresenta as informações. O jogo Lightbot foi utilizado para avaliar a aprendizagem por descoberta, onde acreditava-se ter uma interface simples e objetiva com potencial para proporcionar uma interação exploratória. Mas alguns obstáculos quanto a usabilidade foram relatados, tais como, ajuda do software pouco eficaz e dificuldade das crianças de associar as setas rotatórias ao giro à esquerda ou à direita. Também observou-se maior afinidade e melhor desempenho dos meninos na atividade.

A partir de uma revisão sistemática de literatura, o Scratch aparece com o maior número de utilizações, seguido do Alice, AppInventor, Lego Mindstorm, CS Unplugged, AgentSheet/AgentCubes, MATLAB, Scalable Game Design, Python e Kodu.

Dentre os projetos que tiveram por objetivo atrair jovens para a área de computação, o projeto Introcomp, através de cursos de introdução à Computação e preparatórios para a Olimpíada Brasileira de informática, relata que 30\% dos alunos participantes optaram por um curso na área de Computação motivados pelo projeto.

Outro trabalho, abordou diversos conteúdos relacionados à programação de computadores e eletrônica, por meio de trabalhos em grupos integrados, para quatro alunos do ensino médio. Após um ano de participação no projeto eles passaram a se identificar mais com a área da Computação, passando a utilizar o conhecimento sobre programação para resolver e automatizar a solução de problemas de Física e Matemática.

Do levantamento que investigou a compreensão de profissionais da Computação em relação ao PC, os resultados apontaram que que $64 \%$ dos pesquisados desconhecem o 
V Congresso Brasileiro de Informática na Educação (CBIE 2016)

Anais do XXVII Simpósio Brasileiro de Informática na Educação (SBIE 2016)

PC e sugerem que o termo e habilidades relevantes são pouco conhecidos e compreendidos pelos profissionais, tanto na indústria como na academia.

O trabalho que analisa experiências didáticas que inter-relacionam o PC e a Matemática identifica que Álgebra, Cálculo, Geometria Planar e Aritmética são os conteúdos que têm sido aplicados para o desenvolvimento de habilidades do PC, que linguagens e ambientes visuais de programação são as ferramentas mais utilizadas para este fim e que o público alvo da maioria dos relatos é a educação básica.

Ademais, são levantadas estratégias a serem estabelecidas para a disseminação do PC, como a qualificação de profissionais, a adequação da infraestrutura das escolas, a readequação do currículo escolar e a definição de uma política de implantação do PC.

Com relação a oficina de Computação Desplugada aplicada a professores destacase a disseminação do método, a possibilidade de criar novas atividades ou possíveis extensões, a aplicabilidade para diversos públicos, a possibilidade de tornar as aulas mais atrativas, entre outros, como pontos positivos e as dificuldades pessoais de lidar com mídias e o pouco tempo para a realização das atividades como pontos negativos. Além disso, $89 \%$ dos professores consideraram replicar as atividades nas suas escolas.

Com respeito as correlações entre as competências do PC e os parâmetros curriculares nacionais, destaca-se três competências da matemática com um maior potencial de desenvolvimento conjunto, que são: articulação dos símbolos e códigos; estabelecimento de relações e identificação de regularidades e modelos explicativos e representativos.

Relata-se também que existe uma correlação moderada entre o desempenho dos estudantes no ENEM e a experiência destes em programação. Na análise do teste de PISA com relação aos conceitos do PC, descreve-se que os conceitos de abstração e análise de dados foram cobertos em $100 \%$ e $98,76 \%$ das questões, respectivamente. Decomposição de problemas foi coberto em aproximadamente 39\%, representação de dados em $6 \%$, coleta de dados em quase $4 \%$ e algoritmos e procedimentos em aproximadamente $2 \%$. Automação, paralelismo e simulação não foram abordados.

\section{Considerações Finais}

Este trabalho, fez um mapeamento do PC dos últimos 6 anos, de 2010 a 2015, em 8 principais veículos na área de Informática na Educação no Brasil. É possível observar que a maioria dos projetos realizam práticas de ensino, com o objetivo principal de introduzir conceitos do PC. Estas experiências de ensino são na sua maioria aplicadas ao Ensino Fundamental, utilizando as ferramentas Scratch e "Computação Desplugada".

Os resultados relatam: um aumento do interesse pela área da computação, por todos os públicos trabalhados, inclusive as meninas que foram foco de alguns estudos; maior esclarecimento e/ou conhecimento sobre o tema PC; habilidades comuns entre a Matemática e o PC; propostas de metodologias e formas de avaliar o PC; entre outros. A maioria dos trabalhos tem a perspectiva de continuar os projetos, ampliando a quantidade de alunos e o público-alvo. Assim como incentivam a busca de ferramentas, metodologias e avaliações para o ensino de computação no Brasil, mostrando que o tema PC no país ainda está em expansão. Como projetos futuros, pretende-se ampliar este levantamento para além do Brasil, o que permitirá a correlação entre as metodologias, práticas e ferramentas adotadas no Brasil e no exterior. 
Tabela 1. Relação de Trabalhos

\begin{tabular}{|c|c|c|c|}
\hline ID & Obj. & Ano & Autor(es) \\
\hline 1 & \multirow{18}{*}{ INT } & 2012 & França et al. \\
\hline 2 & & 2012 & Scaico et al. \\
\hline 3 & & 2013 & Andrade et al. \\
\hline 4 & & 2013 & Carvalho et al. \\
\hline 5 & & 2014 & Campos et al. \\
\hline 6 & & 2014 & França et al. \\
\hline 7 & & 2014 & Viel et al. \\
\hline 8 & & 2014 & Wangenheim et al. \\
\hline 9 & & 2015 & Araújo et al. \\
\hline 10 & & 2015 & França et al. \\
\hline 11 & & 2015 & Hinterholz et al. \\
\hline 12 & & 2015 & Paiva et al. \\
\hline 13 & & 2015 & Rabêlo et al. \\
\hline 14 & & 2015 & Rodriguez et al. \\
\hline 15 & & 2015 & Santos et al. \\
\hline 16 & & 2015 & Santos et al. \\
\hline 17 & & 2015 & Schoeffel et al. \\
\hline 18 & & 2015 & Siva et al. \\
\hline$\overline{19}$ & \multirow{2}{*}{ INT, MET } & 2012 & Scaico et al. \\
\hline 20 & & 2013 & França et al. \\
\hline 21 & \multirow{7}{*}{ MET } & 2013 & Gomes et al. \\
\hline 22 & & 2014 & França et al. \\
\hline 23 & & 2015 & França et al. \\
\hline 24 & & 2015 & Morais et al. \\
\hline 25 & & 2015 & Ramos et al. \\
\hline 26 & & 2015 & Silva Neto et al. \\
\hline 27 & & 2015 & Zanetti et al. \\
\hline 28 & MET, LEV & 2015 & Raabe et al. \\
\hline 29 & FER & 2015 & Gomes et al. \\
\hline 30 & \multirow{4}{*}{ FER, ATR } & 2013 & França et al. \\
\hline 31 & & 2014 & Silva et al. \\
\hline 32 & & 2014 & Pereira Júnior et al. \\
\hline 33 & & 2015 & Falcão et al. \\
\hline 34 & FER, ATR, LEV & 2015 & Bombasar et al. \\
\hline 35 & \multirow{2}{*}{ ATR } & 2015 & Meneses et al. \\
\hline 36 & & 2015 & Ramos et al. \\
\hline 37 & \multirow{4}{*}{ LEV } & 2013 & Carvalho et al. \\
\hline 38 & & 2015 & Araújo et al. \\
\hline 39 & & 2015 & Barcelos et al. \\
\hline 40 & & 2015 & Weisshahn et al. \\
\hline 41 & LEV, FOR & 2015 & Farias et al. \\
\hline 42 & \multirow{2}{*}{ LEV, COR } & 2012 & Barcelos et al. \\
\hline 43 & & 2015 & Mestre et al. \\
\hline 44 & FOR & 2014 & Silva et al. \\
\hline 45 & $\overline{\mathrm{CO}}$ & 2015 & R \\
\hline
\end{tabular}

Título

Ensino de Ciência da Computação na Educação Básica: Experiências, Desafios e Possibilidades

Um Relato de Experiências de Estagiários da Licenciatura em Computação com o Ensino de Comp. para Crianças

Proposta de Atividades para o Desenvolvimento do Pensamento Computacional no Ensino Fundamental

Pensamento Computacional no Ensino Médio Mineiro

Organização de Informações via Pensamento Computacional: Relato de Atividade Aplicada no Ensino Fundamental A disseminação do PC na educação básica: lições aprendidas com experiências de licenciandos em computação Introdução à Programação e à Implementação de Processadores por Estudantes do Ensino Médio

Ensino de Computação com SCRATCH no Ensino Fundamental? Um Estudo de Caso

O Ensino de Computação na Educação Básica apoiado por Problemas: Práticas de Licenciandos em Computação

Explorando o pensamento computacional no ensino médio: do design à avaliação de Jogos digitais

Desenvolvimento do PC: Um relato de atividade junto ao Ensino Médio, através do Estágio Supervis. em Comput. III

Uma Experiência Piloto de Integração Curricular do Raciocínio Computacional na Educação Básica

Scratch na produção de recursos interdisciplinares com disciplinas indígena

Pensamento Computacional: transformando ideias em jogos digitais usando o Scratch

Relato sobre o ensino de algoritmos a estudantes do quinto ano do ensino público fundamental

Proposta de atividade para o quinto ano do ensino fundamental: Algoritmos Desplugados

Experiência no Ensino de Pensamento Computacional para Alunos do Ensino Fundamental

Relato de Experiência de Ensino de Comput. no Ensino Fund. em Estágio Supervis. da UPE no Campus Garanhuns

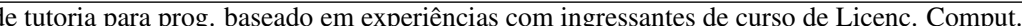

Proposta Metodológica de Ensino e Avaliação para o Desenvolvimento do PC com o Uso do Scratch

O Pensamento Computacional no Ensino Médio: Uma Abordagem Blended Learning

Um modelo colaborativo para a aprendizagem do pensamento computacional aliado à autorregulação

Desafios e oportunidades ao ensino do pensamento computacional na educação básica no Brasil

Processos Participativos de Desenvolvimento de Jogos Digitais em Contextos Escolares

Significação da Aprendizagem Através do PC no Ensino Médio: uma Experiência com Scratch

Uma Abordagem para Inclusão do Licenciado em Computação no Ensino Básico

cas de ensino de Programação de Computadores com Robótica Pedagógica e aplicação de PC Recomendacões para Introdução do Pensamento Computacional na Educação Básica Avaliação de um Jogo Educativo para o Desenvolvimento do Pensamento Computacional na Educação Infantil

Computino: um jogo destinado à aprendizagem de Números Binários para estudantes da educação básica

Análise de ferramentas para o ensino de Computação na Educação Básica

Avaliação de tarefas com uso de tinta digital

Aperta o Play! Análise da Interação Exploratória em um Jogo Baseado em Pensamento Computacional

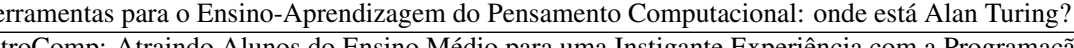

Ensino de Programação para Alunas de Ensino Médio: Relato de uma Experiência

Uma discussão introdut. sobre os desafios e as necessidades à implement. do PC no ensino fund. médio no Brasil

Relações entre o Pensamento Computacional e a Matemática: uma Revisão Sistemática da Literatura

Pensamento Computacional no Brasil: O Estado da Arte

Pensamento Computacional em Sala de Aula: Desafios, Possibilidades e a Formação Docente

ento Computacional e Educação Matemática- Relações para o Ensino de Computação na Educação Básica

Pensamento Computacional: Um estudo empírico sobre as questoes de matemática do PISA

Oficinas Itinerantes de Scratch e Comput. Desplug. para Prof. como apoio ao Ensino de Comput.: Relato de Experiência

Rodrigues et al.
Público $\mid$ Ferramentas

\section{Scratch}

Desplugada

Desplugada

Jogos digitais diverso

Desplugada

Desplugada, Computino, Scratch

IDEBIPide, Portugol, Linguage

Scratch

Desplugada, Scratch

Stencyl

Desplugada

Desplugada

Scratch

Scratch

Desplugada, Scratch, Hora do Códi

Desplugada

Robomind, Scratch, Lego

Gamemaker

Desplug., Robótica PNCA e LightBo

Scratch

App Inventor

NA

Jogos digitais diversos

NA

Scratch

Jogos digitais diversos

Ambiente de prog. S4A, Arduino NA

The Foos

RoboMind, Scratch, Logo, Kodu e Alio

Tinta Digital Lightbot

Linguagem C, Scratch

App Inventor

NA $\quad$ NA

PROFI NA

NA NA

\begin{tabular}{l|l} 
EB & NA \\
\hline ES & NA \\
\hline
\end{tabular}

NA

NA NA

\begin{tabular}{l|l} 
PROFE & Desplugada, Scratch \\
\hline
\end{tabular} 
V Congresso Brasileiro de Informática na Educação (CBIE 2016)

Anais do XXVII Simpósio Brasileiro de Informática na Educação (SBIE 2016)

\section{Referências}

Araújo et al. (2015). Pensamento computacional sob a visão dos profissionais da computação: uma discussão sobre conceitos e habilidades. In Anais do WAlgProg/CBIE.

Barcelos, T. et al. (2015). Relações entre o pensamento computacional e a matemática: uma revisão sistemática da literatura. In Anais do WAlgProg/CBIE.

Barcelos, T. S. and Silveira, I. F. (2012). Pensamento computacional e educação matemática: Relações para o ensino de computação na educação básica. In Anais do WEI.

Bell, T., Witten, I. H., and Fellows, M. (1998). Computer Science Unplugged: Off-line activities and games for all ages.

Bombasar, J., Raabe, A., Miranda, E. M. d., and Santiago, R. (2015). Ferramentas para o ensinoaprendizagem do pensamento computacional: onde está alan turing? In Anais do SBIE.

Carvalho, T. et al. (2013). Uma discussão introdutória sobre os desafios e as necessidades à implementação do pensamento computacional no ensino fundamental e médio no brasil. In Anais do Workshop Escola de Informática Teórica.

Cesar, D. R. (2013). Robótica livre. http: //portal .roboticalivre.org/.

CSTA (2011). Computational thinking - leadership toolkit. https://csta.acm.org/ Curriculum/sub/CurrFiles/471.11CTLeadershiptToolkit-SP-vF.pdf.

Falcão, T. P. and Barbosa, R. (2015). “Aperta o play!”Análise da interação exploratória em um jogo baseado em pensamento computacional. In Anais do SBIE.

Farias, A., Andrade, W., and Alencar, R. (2015). Pensamento computacional em sala de aula: Desafios, possibilidades e a formação docente. In Anais do WAlgProg/CBIE.

França, R. and Tedesco, P. (2014). Um modelo colaborativo para aprendizagem do pensamento computacional aliado à autorregulação. In Anais do SBIE.

Mestre, P. et al. (2015). Pensamento computacional: Um estudo empírico sobre as questoes de matemática do pisa. In Anais do WAlgProg/CBIE.

Papert, S. (1980). Mindstorms: Children, computers, and powerful ideas. Basic Books, Inc.

Raabe, A. et al. (2015). Recomendações para introdução do pensamento computacional na educação básica. In Anais do Workshop Desafios da Computação Aplicada à Educação.

Ramos, F. and Teixeira, L. d. S. (2015). Significação da aprendizagem através do pensamento computacional no ensino médio: uma experiência com scratch. In Anais do WIE.

Rodriguez, C. et al. (2015). Pensamento computacional: transformando ideias em jogos digitais usando o Scratch. In Anais do WIE.

Weisshahn, Y. et al. (2015). Pensamento computacional no brasil: O estado da arte. In Anais do Workshop Escola de Informática Teórica.

Wing, J. M. (2006). Computational thinking. Communications of the ACM, 49(3):33-35. 